ARTICLE

\title{
Surface Landau levels and spin states in bismuth (111) ultrathin films
}

Hongjian Du ${ }^{1, \star}$, Xia Sun ${ }^{1, \star}$, Xiaogang Liu ${ }^{1}$, Xiaojun $\mathrm{Wu}^{1}$, Jufeng Wang ${ }^{1}$, Mingyang Tian ${ }^{1}$, Aidi Zhao ${ }^{1}$, Yi Luo ${ }^{1}$, Jinlong Yang ${ }^{1}$, Bing Wang ${ }^{1} \&$ J.G. Hou ${ }^{1}$

The development of next-generation electronics is much dependent on the discovery of materials with exceptional surface-state spin and valley properties. Because of that, bismuth has attracted a renewed interest in recent years. However, despite extensive studies, the intrinsic electronic transport properties of Bi surfaces are largely undetermined due to the strong interference from the bulk. Here we report the unambiguous determination of the surface-state Landau levels in Bi (111) ultrathin films using scanning tunnelling microscopy under magnetic fields perpendicular to the surface. The Landau levels of the electron-like and the hole-like carriers are accurately characterized and well described by the band structure of the Bi (111) surface from density functional theory calculations. Some specific surface spin states with a large g-factor are identified. Our findings shed light on the exploiting surfacestate properties of Bi for their applications in spintronics and valleytronics.

\footnotetext{
${ }^{1}$ Hefei National Laboratory for Physical Sciences at the Microscale and Synergetic Innovation Center of Quantum Information \& Quantum Physics, Key Laboratory of Strong-Coupled Quantum Matter Physics (CAS), University of Science and Technology of China, Hefei, Anhui 230026, China. * These authors contributed equally to this work. Correspondence and requests for materials should be addressed to B.W. (email: bwang@ustc.edu.cn) or to

J.G.H. (email: jghou@ustc.edu.cn).
} 
$\mathrm{T}$ wo-dimensional (2D) electron materials with honeycomb lattice structure commonly have a band structure with the conduction and valence-band edges at degenerate extrema in momentum space, usually referred to as valleys. The valley degree of freedom of electrons, in addition to the intrinsic charge and spin, has potential to be used as an information carrier in next-generation electronics ${ }^{1-11}$ and optoelectronics ${ }^{12-18}$. Among a variety of appealing materials, bismuth (Bi) has attracted particular attention because of its unique electronic properties both in bulk ${ }^{10,11}$ and surfaces ${ }^{19-29}$. As a heavy element, Bi possesses metallic and spin-split Rashba surface states ${ }^{19,20}$ with a vortical spin texture ${ }^{21,22}$, resulting from the strong spin-orbit coupling (SOC) with the broken space-inversion symmetry ${ }^{20}$. Due to the rhombohedral symmetry in Bi crystal, the Bi (111) surface has a bilayer-terminated structure ${ }^{19}$, forming a buckled honeycomb lattice that leads to valleys in surface-band structure, which is very similar to many other $2 \mathrm{D}$ valley materials with honeycomb lattice ${ }^{1-7,13-18}$. In addition, it has also attracted increasingly study interest in Bi films or bilayers both theoretically $y^{30-32}$ and experimentally ${ }^{33-36}$, owing to the interesting one-dimensional topological edge state, that is, quantum spin Hall state ${ }^{37,38}$.

To explore the applications of surface-state valleys, it demands robust valley properties in a material. Theoretical calculations suggested the transition of $\mathrm{Bi}$ (111) bilayers going from semiconductor to semimetal with the increase of thickness with the crossover thickness around four or five bilayers ${ }^{31,39}$. Bi (111) films with thickness over four bilayers exhibit surface states ${ }^{39}$. The surface-state bands near the Fermi level $\left(E_{\mathrm{F}}\right)$ form electron pockets (EPs) around the $\bar{\Gamma}$ point and the $\bar{M}$ point, and the hole pockets (HPs) along the $\bar{\Gamma} \rightarrow \bar{M}$ directions in films thicker than 10 bilayer $^{39}$. These multivalley states were characterized in Bi films and $\mathrm{Bi}$ crystal by angle-resolved photoemission spectroscopy (ARPES $)^{21-27}$. Their significant contributions to the conductance were also observed in Bi (111) thin films by the transport measurements ${ }^{28,29}$. However, the intrinsic electronic transport
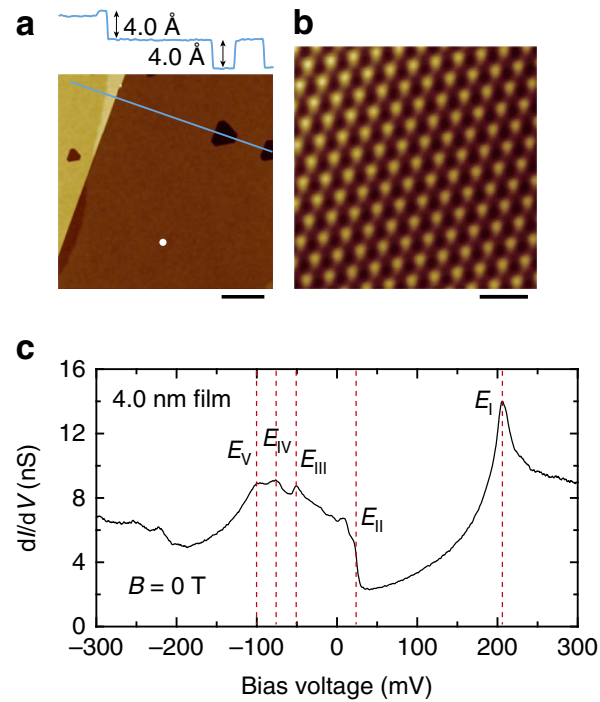

properties of $\mathrm{Bi}$ (111) surface have not been fully determined. For instance, it was reported that the films thicker than six bilayers show thickness-dependent conductivity, which is ascribed to a coherent bulk-surface coupling ${ }^{29}$. To understand the valley properties of $\mathrm{Bi}$ (111) surface for its potential applications in valleytronics $^{1-7}$, it is necessary to single out the electronic transport properties of the surface states. The measurements of the Landau levels (LLs) of the surface-state EPs and the HPs, especially for the relatively complicated surface-band structures of Bi surfaces ${ }^{19}$, can help to determine the contribution of the metallic surface states to the conduction.

We here focus our investigation on the surface-state valleys of Bi (111) surface. We fabricate ultrathin Bi (111) films with various thicknesses grown on $\mathrm{Si}(111)-(7 \times 7)$ and measured under the magnetic field using scanning tunnelling microscopy (STM) and spectroscopy (STS). This enables us to unambiguously identify the LLs of the surface states of the Bi (111) surface. Our experimental results are in good agreement with the density functional theory (DFT) calculations. Moreover, it is observed that the out-of-plane spin states undergo spin polarization as the result of the Zeeman splitting, which may further cause the lift of the degeneracy of certain surface-state valleys.

\section{Results}

STM and STS measurements of a film with thickness of $4.0 \mathrm{~nm}$. Figure 1a shows a representative large-scale image of a $4.0-\mathrm{nm}$ thick Bi film grown on $\mathrm{Si}(111)-(7 \times 7)$. The film has a typical step height of about $4.0 \AA$ and the hexagonal structure with the lattice constant of about $4.5 \AA$ (Fig. 1b), indicating its orientation along the (111) direction ${ }^{19,40}$. Figure $1 \mathrm{c}$ shows a typical $\mathrm{d} I / \mathrm{d} V$ spectrum obtained from the 4.0-nm film. Several features are labelled as $E_{i}$ $(i=\mathrm{I}, \mathrm{II} \ldots, \mathrm{V})$ according to their energy positions. Figure $1 \mathrm{~d}$ gives the $\mathrm{d} I / \mathrm{d} V$ spectra from the film measured at different magnetic field strengths. The field is perpendicular to the sample surface. The LLs show up when the field is larger than $6 \mathrm{~T}$.

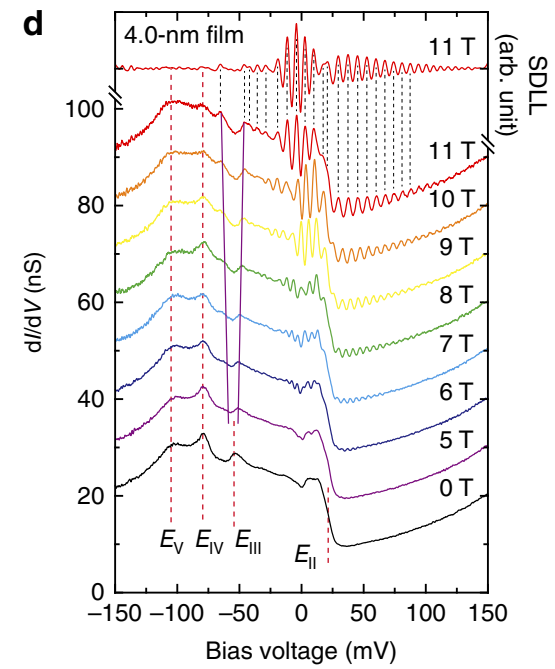

Figure 1 | Electronic properties and Landau levels of Bi (111) surface. (a) A large scale STM image (acquired at $-500 \mathrm{mV}$ and $10 \mathrm{pA}$ ), scale bar, $100 \mathrm{~nm}$. The line profile shows the step height of $4.0 \AA$ for the Bi bilayer structure. (b) Atomically resolved STM image (acquired at $-50 \mathrm{mV}$ and $500 \mathrm{pA}$ ) of Bi (111) film of $4.0 \mathrm{~nm}$ thick, scale bar, $1 \mathrm{~nm}$. (c) Typical $\mathrm{dl} / \mathrm{dV}$ spectrum obtained at centre regions of a large terrace (white dot in a), acquisition conditions: $-300 \mathrm{mV}$ and $4.0 \mathrm{nA}$, with a sinusoidal modulation of $2 \mathrm{mV}$ by root mean square (r.m.s.), at magnetic field strength $B=0 \mathrm{~T}$. The red dashed lines indicate the energy positions of several features, labelled as $E_{i}(i=l, I \ldots, V)$. (d) $\mathrm{d} l / \mathrm{d} V$ spectra recorded in different magnetic field strength, acquired at $-150 \mathrm{mV}$ and $4.0 \mathrm{nA}$ with a sinusoidal modulation of $0.5 \mathrm{mV}$ (r.m.s.). All of the spectra are averaged over 10 repeated measurements. The topmost spectrum is the second derivative of the Landau-level (SDLL) spectrum obtained at $11 \mathrm{~T}$, where the LL peaks are more clearly shown by eliminating the background in the $\mathrm{d} / \mathrm{d} V$ spectrum. The spectra are shifted vertically for clarity. The pink lines around the $E_{\text {III }}$ indicate the peak splitting with the increase of the field. The black dashed lines indicate the peak positions in the SDLL spectrum corresponding to the ones in the $d / / d V$ spectrum. 
A distinct peak splitting at the $E_{\mathrm{III}}$ can be observed with the increase of the magnetic field, and its implication will be discussed later. We use the second derivative of the Landaulevel (SDLL) spectra ${ }^{41}$, that is, $-\frac{\mathrm{d}^{2}}{\mathrm{~d} V^{2}}(\mathrm{~d} I / \mathrm{d} V)$, to identify the LL peak positions in further analysis. For instance, the topmost spectrum displays the SDLL spectrum obtained at $11 \mathrm{~T}$.

Analysis of LLs as a function of magnetic field. The SDLL pattern for the 4.0-nm-thick film is shown in Fig. 2a, where the field is swept from 8.0 to $11.0 \mathrm{~T}$ with the step of $0.02 \mathrm{~T}$. We can extract two sets of fan diagrams from the overlapped SDLL pattern. According to their positive/negative slopes (brown and cyan lines in Fig. 2a), the fan diagrams are associated with the electron-like or hole-like carriers, respectively. The linearly fielddependent LL peaks in each set are almost equally spaced, suggesting parabolic-like dispersions of the carriers. Therefore, the LLs can be approximately described by $E_{n}=\varepsilon_{0} \pm(n+\gamma) \hbar \omega_{c}$, where $E_{n}$ is the energy position of the $n$th LLs, $n$ the LL index, $\varepsilon_{0}$ the energy minimum of EP or maximum of HP, $\gamma$ the Onsager phase, $\hbar$ the reduced Plank constant, $\omega_{\mathrm{c}}$ the cyclotron frequency, that is, $\omega_{\mathrm{c}}=e B / m^{*}, e$ the electron charge, $B$ the magnetic field strength, and $m^{*}$ the cyclotron effective mass of carriers. Signs ' + ' and ' - ' are applied to the electron-like or the hole-like carriers, respectively.

Analogous to the traditional measurements of Shubnikov-de Haas oscillations, the tunnelling magnetoconductance oscillations ${ }^{42}$ can be obtained at every bias voltage, $V_{\mathrm{b}}$. Figure $2 c$ gives an example of the aperiodic oscillations in the cut-line at
$+30 \mathrm{mV}$, where the peaks correspond to the same constant energy cross-sectional area (CEC area, $A$ ), following the Onsager relation $A=(2 \pi e / \hbar)(n+\gamma) B$. The LL indexes for the HP are easily obtained by adopting the topmost $\mathrm{LL}$ as $n_{\mathrm{h}}=0$. In the determination of the LL indexes for the EP, we use the Onsager relation to fit the LL index $n_{\mathrm{e}}$. At a given energy, for example, see the cut-line at $30 \mathrm{mV}$ in Fig. $2 \mathrm{c}$, the LL peaks correspond to the same value of $A$. The LL indexes fitted by the least square method are obtained and labelled in Fig. 2a. The fitting value of $\gamma$ is about $1 / 2$ and slightly dependent on energy. Tentatively assuming the parabolic dispersion for the EP and HP, we have $e V_{\mathrm{b}}-\varepsilon_{0}= \pm \hbar^{2} k^{2} / 2 m^{\star}$, where $k$ is the wave vector. In the momentum space, $A=\pi k^{2}$. Then, we have $A= \pm\left(2 \pi m^{\star} / \hbar^{2}\right)\left(e V_{\mathrm{b}}-\varepsilon_{0}\right)$, which describes the linear dependence of the CEC area $A$ on energy, $e V_{\mathrm{b}}$.

Similar to the analysis used in Shubnikov-de Haas oscillations, the SDLL pattern is replotted as a function of $1 / B$ (Fig. $2 b$ ), and the oscillations in the cut-line now give the periodicity of $\Delta\left(B^{-1}\right)$ (Fig. 2d). The oscillation frequency, $f_{B}=1 / \Delta\left(B^{-1}\right)$, is obtained by Fourier transform (FT) of every cut-line, and associated with the CEC area by $A=(2 \pi e / \hbar) f_{B}$. The FT pattern of the $1 / B$ plot is shown in Fig. 2e, which well reflects the linear dependence between $A$ and $V_{\mathrm{b}}$ both for the LLs of the EP and HP, respectively. Alternatively, using the field strength of the marked points at the LL peaks (red dots Fig. 2a), the CEC areas can be obtained by adopting the LL indexes using the Onsager relation. In the overlapped region of the SDLL pattern we have selected the cross points that belong to the LLs both from the EP and HP. For the LLs above $40 \mathrm{mV}$ (only from the EP), the points at the a

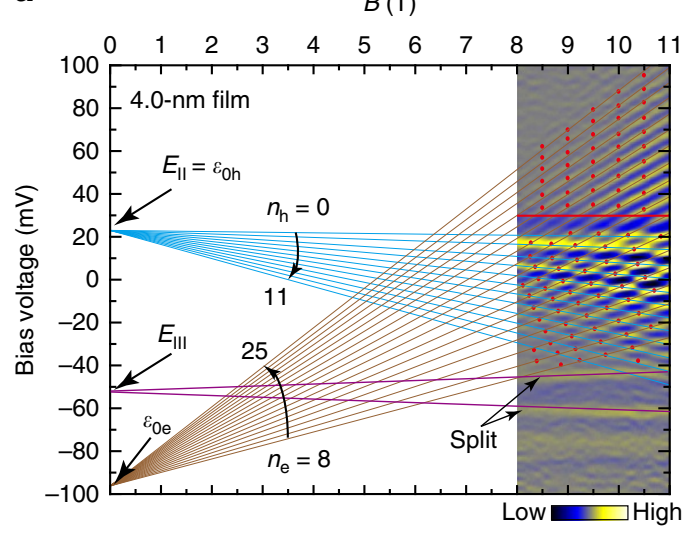

b

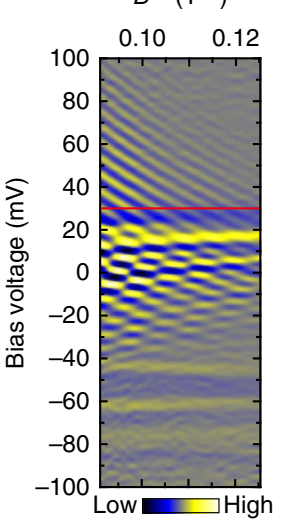

d

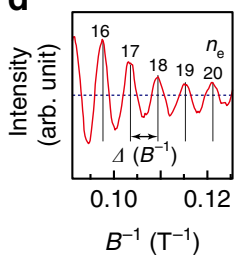

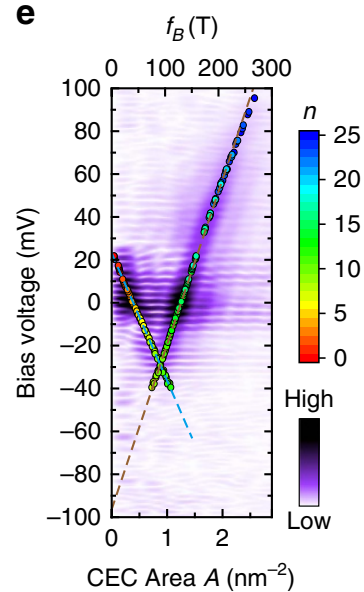

CEC Area $A\left(\mathrm{~nm}^{-2}\right)$

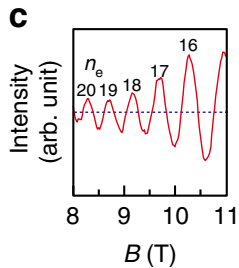

Figure 2 | Analysis of the Landau levels. (a) The second derivative of the Landau-level (SDLL) pattern of the 4.0-nm-thick film as a function of $B$. Acquisition conditions: $-150 \mathrm{mV}$ and $4 \mathrm{nA}$, modulation of $1.0 \mathrm{mV}$ by root mean square (r.m.s.). The brown and cyan lines are the fan diagrams of the Landau levels (LLs) for the electron-like and hole-like carriers, respectively, extracted from the pattern. The fitted LL indexes $\left(n_{\mathrm{e}}\right.$ or $\left.n_{\mathrm{h}}\right)$, and the extrapolated electron pocket minimum $\left(\varepsilon_{\mathrm{Oe}}\right)$ and hole pocket maximum $\left(\varepsilon_{\mathrm{Oh}}\right)$ are labelled, where the subscripts ' $\mathrm{e}$ ' and ' $\mathrm{h}$ ' refer to the electron-like and the hole-like carriers, respectively. The peak splitting at the $E_{\| I I}$ is also indicated by the pink lines. (b) Replotted SDLL pattern as a function of $1 / B$. (c) Profile of the cutline at $30 \mathrm{mV}$ in $\mathbf{a}$. (d) Profile of the cut-line at $30 \mathrm{mV}$ in $\mathbf{b}$, showing the periodicity of $\Delta\left(B^{-1}\right)$. (e) Fourier transform pattern of oscillation frequency $f_{B}$ (top-coordinate) or constant energy cross-sectional (CEC) area $A$ (bottom-coordinate), superposed with colour circles that are obtained from the selected points (red dots in a) of LL peaks using the Onsager relation. The dashed lines (brown and cyan) in e are linear fits of the data (colour circles) for the electron pocket and the hole pocket, respectively. The colour bars in $\mathbf{e}$ indicate the LL index of the calculated points using the selected points in a and the relative intensity of the Fourier transform pattern, respectively. 
magnetic field strength of 8.5, 9.0, 9.5, 10.0, and $10.5 \mathrm{~T}$ are chosen, respectively. The CEC areas obtained from the individual points are superposed in Fig. 2e, denoted by coloured circles. Obviously, these data from the EP and HP can be well-fitted linearly, respectively, shown by the dashed lines in brown and cyan.

Comparing DFT calculations with experimental results. Our experimental observations can be well interpreted by the electronic structures of the $\mathrm{Bi}$ (111) surface obtained from the DFT calculations. Figure 3a shows the calculated band structure using a thin-film modelled with 10 Bi bilayers, corresponding to a 4.0-nm-thick film. The two branches in brown and cyan dominantly contribute to the surface states. Figure $3 \mathrm{~b}$ gives the calculated density of states from these two branches, which well describes the experimentally observed five features in the $\mathrm{d} I / \mathrm{d} V$ spectra. For comparison with the experimental results, we set the $E_{\mathrm{F}}$ to $+18.0 \mathrm{meV}$, that is, the calculated electronic structure is shifted downwards by $18 \mathrm{meV}$. Except the step-like feature at the $E_{\mathrm{II}}$ (HP maxima, $\varepsilon_{0 \mathrm{~h}}$ ), other features appeared as peaks are from the van Hove singularities (VHSs) ${ }^{43}$, that is, the saddle points $\left(E_{\mathrm{I}}, E_{\mathrm{III}}\right.$ and $\left.E_{\mathrm{IV}}\right)$ or the plateaus $\left(E_{\mathrm{V}}\right)$ in the band structure, as shown by the magnified surface-state band structure in Fig. 3c. This band structure can well explain the overlap of the two LL sets in our experiment. The EP does not drop to the extrapolated minimum $\left(\varepsilon_{0 \mathrm{e}}\right)$, but forms a Mexican-hat-like bottom around $-50 \mathrm{meV}$. Additional tiny pockets, with a depth of $\delta \sim 4 \mathrm{meV}$ with respect to the $E_{\mathrm{III}}$, can be seen at the EP bottom in the $\bar{\Gamma} \rightarrow \bar{M}$ directions. It is noted that the EP and HP are both deviated from the ideal parabolic dispersion, appearing as hexagonal and ellipse-like shapes. In comparison, the calculated Fermi contour is in good accordance with the ARPES results from Bi crystal and Bi films ${ }^{23-27}$ (Supplementary Fig. 1, Supplementary Note 1). The CEC areas of the energy-overlapped EP and HP are calculated and shown in Fig. 3d, which are in good agreement with our experimental results.

Dependence of the surface states on film thickness. It has been theoretically predicted that the surface-state band structure of $\mathrm{Bi}$ films has just a slight dependence on the thickness when the films are thicker than 10 bilayers $^{39}$. The typical $\mathrm{d} I / \mathrm{d} V$ spectra taken from several samples with different thicknesses at $0 \mathrm{~T}$ are shown in Fig. 4a, and replotted in Fig. $4 \mathrm{~b}$ by aligning them according to the $E_{\mathrm{II}}$ of the $4.0-\mathrm{nm}$-thick film. It is observed that the main features in $\mathrm{d} I / \mathrm{d} V$ may shift upward or downwards with respect to the $E_{\mathrm{F}}$ in different samples and even in different sites of the same sample (Supplementary Figs 2-4). Such shifts are attributed to the variations of the Fermi energy in different samples or even local sites affected by the STM tip ${ }^{44}$. Despite the energy shift the

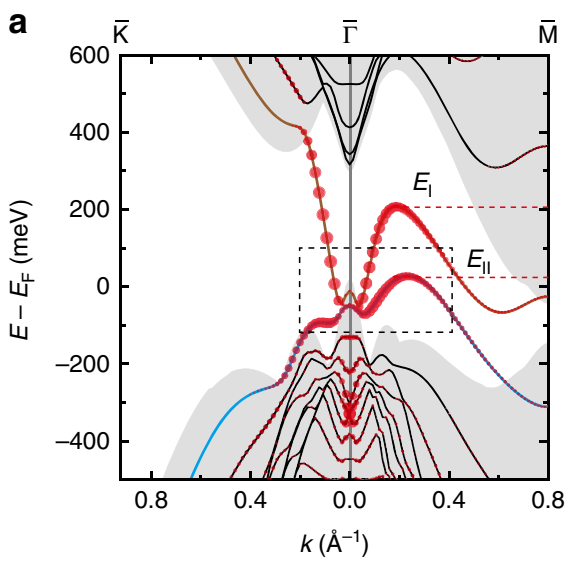

b

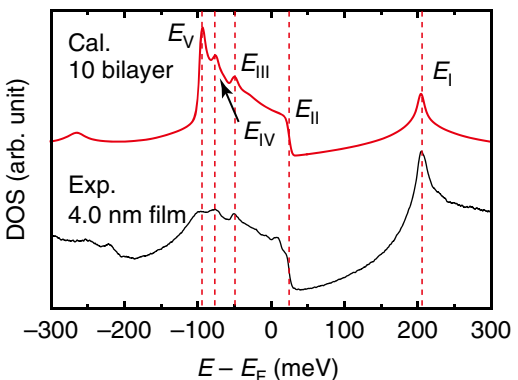

C

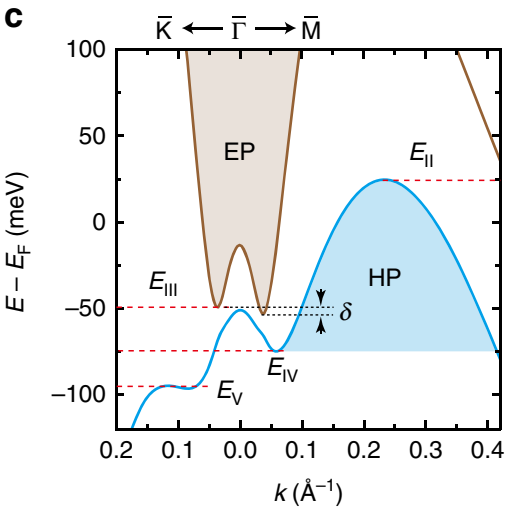

d

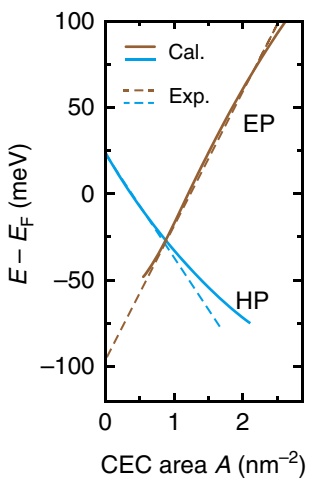

Figure 3 | Calculated electronic structure of Bi (111) surface. (a) Calculated band structure (solid lines) of Bi (111) surface using a model of 10 Bi bilayers. The surface-state weightings are denoted by the relative size of the red dots. The shadow background is the calculated projection of bulk band structure. The calculated electronic structure is shifted downward by $18 \mathrm{meV}$ for comparing with the experimental results. (b) Calculated density of states (DOS, solid red curve) with a Gaussian broadening of $3.0 \mathrm{meV}$, in comparison with the experimental $\mathrm{d} / / \mathrm{d} V$ spectrum (in black) of the 4.0-nm-thick film. (c) Magnified band structure around the $\bar{\Gamma}$ point of the dashed rectangle region in $\mathbf{a}$. The dashed red lines correspondingly indicate the energy positions of $E_{i}(i=\mathrm{I}, \mathrm{II} \ldots, \mathrm{V})$, and the dashed black lines in $\mathbf{c}$ indicate the depth, $\delta$, of the additional tiny pockets with respect to the $E_{I I I}$. (d) Calculated constant energy cross-sectional (CEC) areas of the electron pocket (EP) and the hole pocket (HP) against energy, given by the solid lines in brown and cyan, in comparison with the fitted lines from the experimental data of the 4.0-nm-thick film, given by the dashed lines in brown and cyan. 


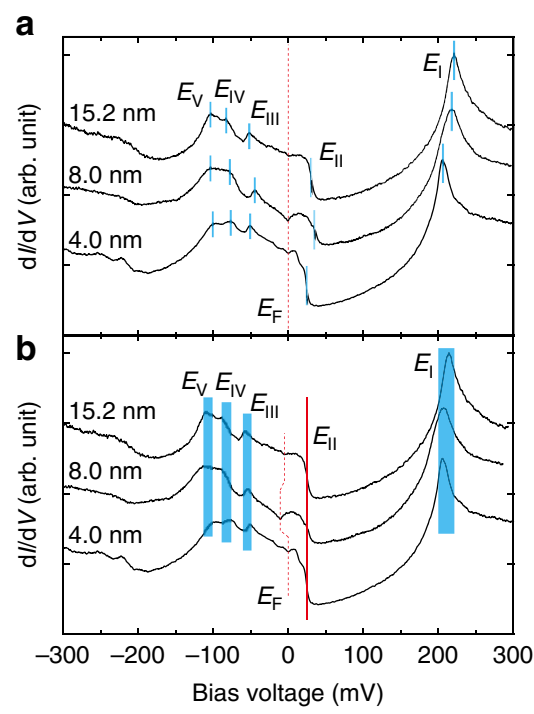

C

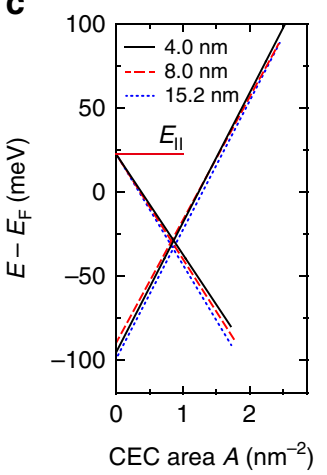

d
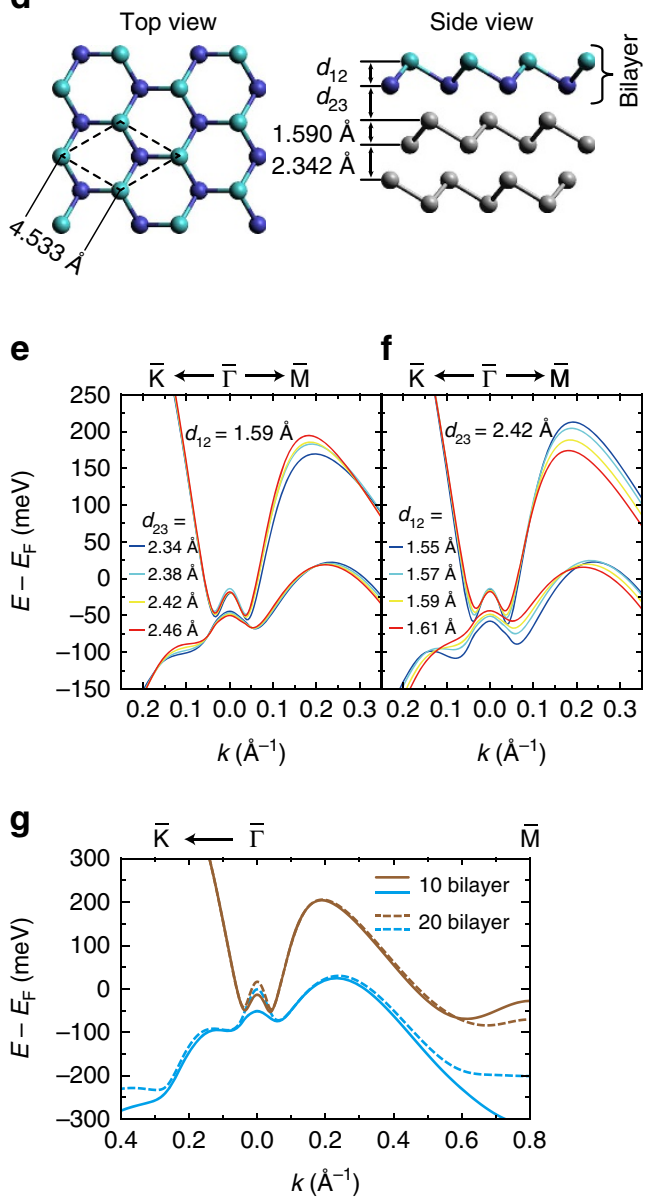

Figure 4 | Dependent aspects of the surface states. $(\mathbf{a}, \mathbf{b})$ Original and aligned spectra obtained from films with different thicknesses, showing that the relative intervals of the features are just slightly dependent on the film thickness. The spectra in $\mathbf{b}$ are aligned according to the $E_{\| \prime}$ of the 4.0 -nm-thick film, as labelled by solid red line. The $E_{\mathrm{F}}$ is marked by the dashed red lines. The energy positions of other features are marked by solid cyan lines (before alignment in $\mathbf{a}$ ) and cyan bars (after alignment in $\mathbf{b}$ ). The spectra in $\mathbf{a}$ and $\mathbf{b}$ are shifted vertically for clarity. (c) Constant energy cross-sectional (CEC) areas obtained from fitting the Landau levels of the samples with different thicknesses, respectively, aligned according to the $E_{\|}$of the 4.0-nm-thick film.

(d) Structural model and parameters used in the calculations. (e,f) Calculated surface-state band branches by fixing $d_{12}$ but varying $d_{23}$, and by fixing $d_{23}$ but varying $d_{12}$, respectively, for the terminated Bi bilayer. (g) Calculated surface-state band branches of 10 (solid lines) and 20 (dashed lines) Bi bilayers.

intervals between the features just slightly vary, indicating their surface-dominant nature and weak dependence on film thickness. Figure $4 \mathrm{c}$ shows the $\mathrm{CEC}$ areas obtained from the LL measurements for the films with different thicknesses (Supplementary Fig. 5), which also reflects the almost independent behaviours of the EP and HP on film thickness. Considering the shift of the $E_{\mathrm{F}}$ in different samples, we plot the fitting lines by aligning them according to the $E_{\mathrm{II}}$ of the $4.0-\mathrm{nm}$ thick film. It was recently reported the existence of semimetal-tosemiconductor transition in thicker film of about 180 bilayers ${ }^{45}$, however, it is quite doubted by just measuring the bands at $\bar{\Gamma}$ point without having detailed information from the $\bar{M}$ point if the $E_{\mathrm{F}}$ shift is taken into consideration. Actually, in ref. 45 the changes of the measured CEC areas for the EP and HP in different thicknesses can be well explained by the $E_{\mathrm{F}}$ shift (Supplementary Table 1).

In our calculations, we used the lattice parameters of bulk crystal with a rhombohedral A7 structure ${ }^{40}$ for the inner $\mathrm{Bi}$ bilayers, which gives the distance of $1.590 \AA$ between the atom layers of each bilayer and the distance of $2.342 \AA$ between the neighbour bilayers along the (111) direction, as shown in Fig. 4d. We find that the band structure is much sensitive to the lattice parameters of the terminated Bi bilayer, as shown in Fig. 4e,f. We adopt the parameters of $d_{12}=1.570 \AA$ and $d_{23}=2.420 \AA$ in the terminated $\mathrm{Bi}$ bilayer, for these parameters give consistent calculated results with our experimental observations.

We also compared the calculated surface-state bands of 20 bilayers with the ones of 10 bilayers, as shown in Fig. 4g. It is seen that except the states around $\bar{\Gamma}, \overline{\mathrm{K}}$ and $\overline{\mathrm{M}}$ points the surface-state bands of the two films are almost overlapped, which support the observed thickness-independent features. The non-overlapped states around the high symmetric points are ascribed to the hybridization of the quantum well states (QWSs) ${ }^{26,46}$.

Zeeman splitting of spin states at the $\boldsymbol{E}_{\mathrm{III}}$. We observed the peak splitting at the $E_{\mathrm{III}}$ in all of the three films, as shown in Fig. $5 \mathrm{a}-\mathrm{c}$. The peak splitting is attributed to the Zeeman coupling under non-zero magnetic field perpendicular to the surface, which strongly suggests the existence of large out-of-plane spin components at the saddle points of $E_{\mathrm{III}}$ along the $\bar{\Gamma} \rightarrow \mathrm{K}$ directions (Fig. 5d). Because of the spin texture in the Bi (111) surface, the out-of-plane magnetic moments in two adjacent saddle points should be inversely oriented ${ }^{21}$ (Fig. 5e,f), similar to other related 
a

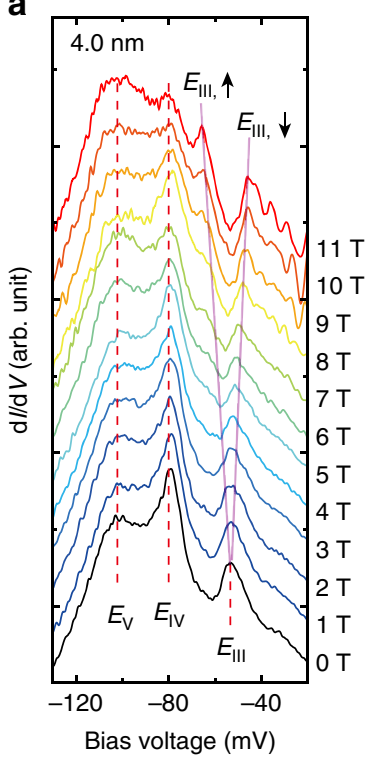

b

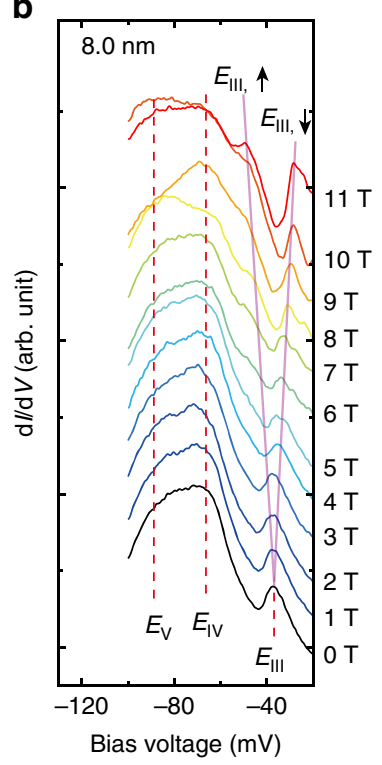

c
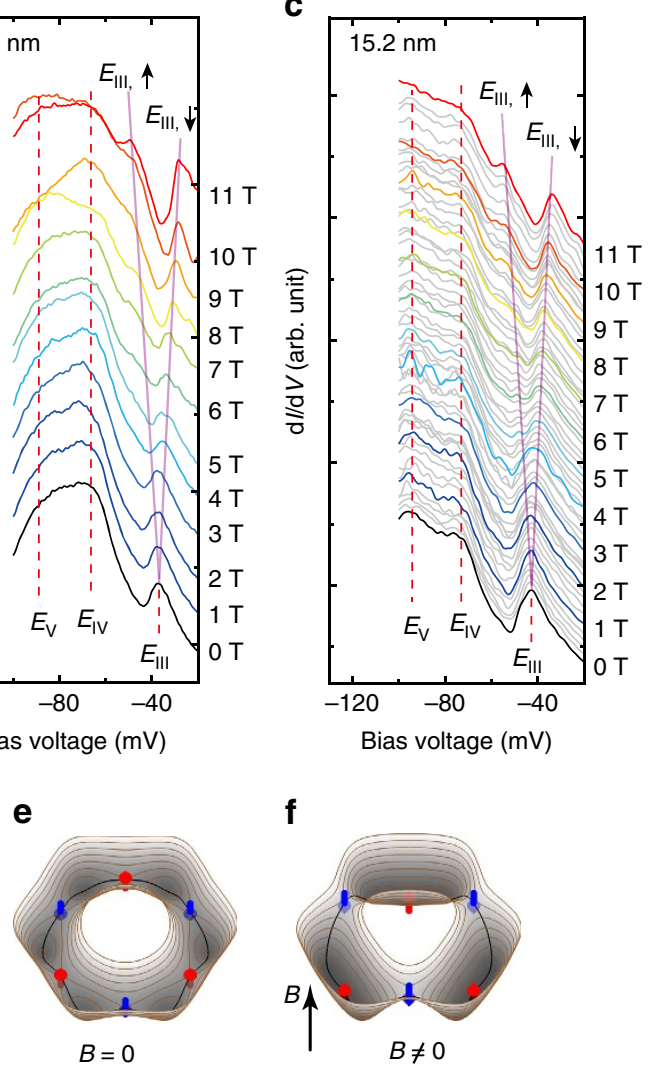

f

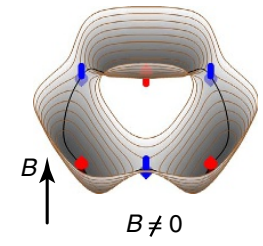

g

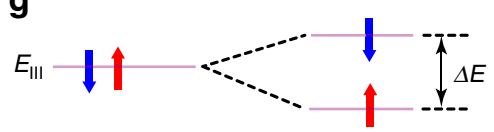

Figure 5 | Spin splitting of the surface states at the $\boldsymbol{E}_{\mathbf{I I I}} \cdot(\mathbf{a}-\mathbf{c}) \mathrm{d} / \mathrm{d} V$ spectra of $B i$ (111) films with thicknesses of $4.0,8.0$, and $15.2 \mathrm{~nm}$, obtained under the labelled magnetic field strengths. Acquisition conditions: $-150 \mathrm{mV}$ and $4.0 \mathrm{nA}$ and modulation of $0.5 \mathrm{mV}$ by root mean square (r.m.s.) in a, $-100 \mathrm{mV}$ and $1.0 \mathrm{nA}$ and modulation of $1.0 \mathrm{mV}$ (r.m.s.) in $\mathbf{b}$, and $-100 \mathrm{mV}$ and $2.0 \mathrm{nA}$ and modulation of $1.0 \mathrm{mV}(\mathrm{rms})$ in $\mathbf{c}$. The spectra in grey are shown with an interval of $0.2 \mathrm{~T}$ in $\mathbf{c}$. The peak splitting at the $E_{\mathrm{III}}$ is marked by the pink lines, but the features at the $E_{\mathrm{IV}}$ and $E_{\mathrm{V}}$ do not have obvious splitting (dashed red lines). The spectra are shifted vertically for clarity. (d) Three-dimensional plot of the band structure around the $\bar{\Gamma}$ point, with labelled saddle points at the $E_{I I I}$ and $E_{I V}$ and the plateau points at the $E_{\mathrm{V}}$. (e,f) Schematic drawing of the out-of-plane spin states at the saddle points of $E_{\| I I}$ in the momentum space at $B=0 \mathrm{~T}$, and the spin splitting at $B \neq 0 \mathrm{~T}$. ( $\mathbf{g}$ ) Energy diagram of the spin degeneracy of the $E_{11}$ at $B=0 \mathrm{~T}$ and the spin splitting of $\Delta E$ at $B \neq 0 \mathrm{~T}$. The red and blue arrows denote spin-up and spin-down, respectively.

systems ${ }^{47-49}$. At $11 \mathrm{~T}$, the splitting energy, $\Delta E$, is about $21 \mathrm{meV}$ in all of the three samples, as illustrated in Fig. 5g. Using $\Delta E=g_{\text {eff }} \mu_{\mathrm{B}} B$, where $\mu_{\mathrm{B}}=e \hbar / 2 m_{0}$ is the Bohr magneton and $m_{0}$ is the rest mass of electron, we get an almost thicknessindependent effective g-factor, $g_{\text {eff }} \approx 33 \pm 1$, which is about half of the value for the hole-like carriers in bulk bismuth ${ }^{50}$. This relatively large $g$-factor could be related to the strong SOC ${ }^{19}$ and the $E_{\mathrm{III}}$ VHS states locating at the turning points from the surface states to the QWSs ${ }^{26,46}$. As a result of the Zeeman splitting, spinpolarized valleys may even be produced at alternate $E_{\mathrm{III}} \mathrm{VHS}$ states when the states are deeper than the minima of the tiny pockets at a high enough magnetic field (Fig. 5f). The much large $g$-factor may guarantee the valleys deeper enough over the depth of $\delta$. It is noticed that the VHS states at the $E_{\text {III }}$ have well determined energy and momentum. We did not observe the Zeeman splitting in individual LLs, unlike the observation of the Zeeman splitting of LLs in other $2 \mathrm{D}$ system $^{51}$ and in bulk $\mathrm{Bi}^{11,50,52}$. This can be understood by considering that the top and the bottom surfaces of the thin films should have degenerate opposite spin vectors $^{22,53}$, while the STM measurements can just detect the top surface of the films.

\section{Discussion}

From the experimentally measured CEC areas (Fig. 4c), we get the estimated electron- and hole-carrier densities to be $D_{\mathrm{e}}=3.13$ $\times 10^{12}$ and $D_{\mathrm{h}}=5.82 \times 10^{12} \mathrm{~cm}^{-2}$ at the $E_{\mathrm{F}}$ from the $4.0-\mathrm{nm}$ film. In the estimation, we include the sixfold valley degeneracy for HP, but do not include the spin degeneracy both for the EP and HP after considering the fact that the degenerate spin should be separately at the top and the bottom surface in the thin films ${ }^{2,53}$. These estimated carrier densities are in good agreement with the previous experimental values of $D_{\mathrm{e}}=2.75 \times 10^{12}$ (ref. 54) and $D_{\mathrm{h}}=8 \times 10^{12} \mathrm{~cm}^{-2}$ (ref. 55) obtained in $\mathrm{Bi}$ thin films. Such agreements may allow us to suggest that the bottom surface (interface) may not obviously contribute to the conduction, possibly ascribing to the strong scattering at the interface. It is also noticed that our estimated values are just about half of those estimated from the ARPES results by Ast and Höchst ${ }^{23}$. This should be attributed from the inclusion of the spin degeneracy in their calculations, since our measured CEC areas for the EP and HP compare well with the ARPES result (Supplementary Fig. 1, Supplementary Table 1). This observation can be important and useful for the measurement 
of the $2 \mathrm{D}$ electron transport property solely from the top surface in the thin films supported on substrates, which is a highly concerned issue in the study of Bi films ${ }^{28,30-36,56}$. In addition, we also expect that $\mathrm{Bi}(111)$ thin films may be suitable for the spin transport measurement by using the energy- and momentumdetermined spin-polarized $E_{\mathrm{III}}$ states (Fig. 5).

Using the fitting slopes of the CEC area against the energy (Fig. 4c), we obtain cyclotron effective masses of the electron- and hole-like carriers, $m_{\mathrm{e}}{ }^{*} \sim(0.16 \pm 0.02) m_{0}$ and $m_{\mathrm{h}}{ }^{*} \sim$ $(0.19 \pm 0.03) m_{0}$. It is noticed that in the single crystal $\mathrm{Bi}(111)$ surface the ARPES results gave the effective masses of $m_{\mathrm{e}}^{*}=(0.22 \pm 0.04) m_{0}$ for the electron-like carrier along the $\bar{\Gamma} \rightarrow \overline{\mathrm{M}}$ direction ${ }^{23}$, and $m_{\mathrm{h}} x^{*}=1.2 m_{0}$ along the $\bar{\Gamma} \rightarrow \overline{\mathrm{M}}$ direction and $m_{\mathrm{h} y}{ }^{*}=0.032 m_{0}$ along the $\bar{\Gamma} \rightarrow \overline{\mathrm{K}}$ direction for the holelike carrier ${ }^{57}$. The masses obtained from the LL measurement should reflect the average values over the masses at different $k$ points due to the dispersion of the EP and HP deviated from the ideal parabolic dispersion. The effective mass of $m_{\mathrm{e}}{ }^{*} \sim 0.16 m_{0}$ in our experiment is still consistent with the value of the single crystal surface, as the EP just slightly deviates from the parabolic dispersion. Due to the ellipse-like cross-sectional shape for HP in momentum space, we get approximately an average value of $\sim 0.20 m_{0}$ by $\left(m_{\mathrm{h} x}{ }^{*} m_{\mathrm{h} y}{ }^{*}\right)^{1 / 2}$. Obviously, the measured cyclotron effective masses from the LLs in our experiment are consistent with the results from the ARPES measurements.

These relatively large values of the effective masses can be used to explain why we can only observe the LLs under relatively high magnetic fields. The full-width at half maximum (FWHM) of the LL peaks is about $4.5 \mathrm{meV}$. Since the STM instrumental broadening is smaller than $0.15 \mathrm{meV}$ (Supplementary Fig. 6), the finite-temperature broadening of $3.5 k_{\mathrm{B}} T \sim 1 \mathrm{meV}$, and the used modulation of $0.5 \mathrm{mV}$ (root mean square (r.m.s.)), we can conclude that the observed line width is dominantly contributed by the intrinsic width of LLs. The observed FWHM thus leads to an estimated lifetime of the carriers, $\tau \sim 0.2 \mathrm{ps}$. With the criteria $\omega_{\mathrm{c}} \tau>1$, a lower limit field of about $6 \mathrm{~T}$ is obtained. Only above this field can the LL peaks be seen, in agreement with our observations (Fig. 1d, and Supplementary Fig. 5) and the theoretical suggestion ${ }^{58}$. Moreover, the quality of the films is also important to observe the LLs, since the LL peaks may be suppressed when the range of disorder is comparable to the magnetic length $l_{B} \sim(\hbar / e B)^{1 / 2}$ (ref. 44). Actually, the LLs can be observed at relatively large terraces, but become less pronounced at or near step edges in our experiment (Supplementary Fig. 4). We can also get an estimated carrier mobility of about $1.7 \times 10^{3}$ $\mathrm{cm}^{2} \mathrm{~V}^{-1} \mathrm{~s}^{-1}$ and a $2 \mathrm{D}$ conductivity of $2.4 \times 10^{-3} \Omega^{-1} \square^{-1}$ contributed by the EP and HPs from the Bi (111) top surface. The conductivity is much comparable to the almost temperatureindependent conductivity of $\sim 1.5 \times 10^{-3} \Omega^{-1} \square^{-1}$ obtained in the Bi film of six bilayers ${ }^{29,56}$. The slight deviation may be due to the existed scattering by the steps in the films used in the transport measurements ${ }^{29,56}$, while our estimation is obtained in a more ideal terrace from the STM measurements.

From our experimental and theoretical results, the EP and HP are quite robust against the change of film thickness from 4.0 to $15.2 \mathrm{~nm}$, even though a certain degree of thickness dependence near the high symmetric points due to the hybridization with QWSs is observed. Considering the fact that the metallic surface states dominantly contribute to the transport properties for films thinner than 20 bilayers $^{29}$, and probably just from the top surface, one may make use of the ultrathin Bi films as a playground to investigate the transport property of multivalley surface states, which possesses huge anisotropic HPs around the $E_{\mathrm{F}}$. Moreover, the existence of strong out-of-plane spin components at the saddle points of $E_{\text {III }}$ along the $\bar{\Gamma} \rightarrow \overline{\mathrm{K}}$ direction can be a novel property to produce spin-polarized valley states. We may thus expect that because of the tunable spin-polarized valleys by magnetic field in addition to the unique surface states, the ultrathin $\mathrm{Bi}$ (111) films can indeed find their applications in spintronics and valleytronics ${ }^{1-7}$.

\section{Methods}

Sample preparation and characterization. Our STM experiments were conducted using an ultrahigh vacuum (UHV) low temperature scanning tunnelling microscope (UNISOKU) operated under a magnetic field up to $11 \mathrm{~T}$ perpendicular to the sample surface, and equipped with a sample preparation chamber for film growth. The base pressure of the system was better than $5 \times 10^{-11}$ torr. The Bi (111) thin films were prepared by depositing Bi (99.997\%, MaTeck) with nominal thicknesses of 4.0 and $8.0 \mathrm{~nm}$ at a precalibrated rate of $0.12 \mathrm{~nm} \mathrm{~min}^{-1}$ on $\mathrm{Si}(111)-7 \times 7$ substrates using a Knudsen cell. During the evaporation, the substrates were kept at room temperature. An As-doped $n$-type $\operatorname{Si}(111)$ wafer with resistivity of about 0.001 to $0.005 \Omega \mathrm{cm}$ was used. The miscut angle of the $\mathrm{Si}(111)$ wafer was about $0.1^{\circ}$, resulting in a $\mathrm{Si}(111)$ terrace width of about $200 \sim 400 \mathrm{~nm}$. After the evaporation, the samples were annealed at $380 \mathrm{~K}$ for about $24 \mathrm{~h}$. Such treatment helped to produce relatively large terraces in the $\mathrm{Bi}$ (111) films. To examine the thicknesses and the corresponding properties, another film with a nominal thickness of about $8.0-\mathrm{nm}$ film was prepared, but followed by annealing at a higher temperature of $420 \mathrm{~K}$ for $24 \mathrm{~h}$. This treatment produced islands due to the dewetting of the $\mathrm{Bi}(111)$ film $^{59}$. In the islands, the electronic properties of the $\mathrm{Bi}$ (111) surface could be well correlated to the determined thicknesses by measuring at the island edges (Supplementary Figs 2 and 3). In this way, the uncertainty of the thicknesses of islands should be within 1 or 2 bilayers. The presented results from the film of $15.2 \mathrm{~nm}$ were obtained from a Bi (111) island of about $5 \mu \mathrm{m}$. All of the STM measurements were performed at $4.3 \mathrm{~K}$. The $\mathrm{d} I / \mathrm{d} V$ spectra of the $\mathrm{Bi}$ (111) surface were measured using a lock-in preamplifier, with a sinusoidal modulation of $0.5 \sim 2.0 \mathrm{mV}$ (r.m.s.) at $791 \mathrm{~Hz}$, under various magnetic fields. An electrochemically etched and well-cleaned tungsten tip was used. The sample bias with respect to the tip was used.

The microscope had a thermal drift smaller than $0.5 \mathrm{~nm} \mathrm{~h}^{-1}$ at zero magnetic field at $4.3 \mathrm{~K}$. There was a systematic drift between the tip and the sample about $20 \mathrm{~nm}$ with the increase of the magnetic field from 7 to $11 \mathrm{~T}$ in our microscope. In the measurements of individual spectra, we always waited for the stabilization of the microscope and repositioned the tip sites when the field was changed. While, in the field-sweeping measurements, we compensated the drift and reexamined the tip sites by every interval of $0.5 \mathrm{~T}$, which could keep the spectra acquired almost at the same sites within an accuracy of about $0.5 \mathrm{~nm}$. For the sake of simplicity, we just focus our discussion of the LL spectra obtained from the centre region of terraces larger than approximately $200 \times 200 \mathrm{~nm}^{2}$. Each set of the spectra with varied magnetic field were measured almost at the same site by tracing the tip-sample drift, to avoid site-dependent shift of the spectra.

Theoretical calculations. The calculations of the electronic structure were performed using the all-electron full potential linearized augmented planewave method implemented in WIEN2k (ref. 60). The generalized gradient approximation in PBE form ${ }^{61}$ was used to describe the electronic exchangecorrelation energy functional. The irreducible surface Brillouin zone (SBZ) was sampled via $12 \times 12 \times 1$ with $144 \mathrm{k}$ points. The muffin tin radius $\left(R_{\mathrm{mt}}\right)$ of 2.5 a.u. was used for $\mathrm{Bi}$ atom. The plane-wave cutoff parameter $R_{\mathrm{mt}} K_{\max }$ was chosen to be 9 ( $K_{\max }$ is the magnitude of the largest $\mathbf{K}$ vector). Inside the muffin tins wave functions were expanded in spherical harmonics up to $l_{\max }=12$. Spin-orbit coupling (SOC) was included. The Bi (111) surface was modelled by a slab with a $(1 \times 1)$ unit cell containing 10 bilayers. A vacuum gap of $15 \AA$ was used between the slabs. The lattice constants of bulk crystal with a rhombohedral A7 structure, that is, $a=4.533 \AA$, and $c=11.797 \AA$ at $4.2 \mathrm{~K}$ (ref. 40 ), were used for the inner $\mathrm{Bi}$ bilayers, but the parameters for the outermost bilayer (both at the top and the bottom sides) were adopted by comparing the calculated results with the experimental results.

\section{References}

1. Neto, A. H. C., Guinea, F., Peres, N. M. R., Novoselov, K. S. \& Geim, A. K. The electronic properties of graphene. Rev. Mod. Phys. 81, 109-162 (2009).

2. Rycerz, A., Tworzydło, J. \& Beenakker, C. W. J. Valley filter and valley valve in graphene. Nat. Phys. 3, 172-175 (2007).

3. Akhmerov, A. R. \& Beenakker, C. W. J. Detection of valley polarization in graphene by a superconducting contact. Phys. Rev. Lett. 98, 157003 (2007).

4. Xiao, D., Yao, W. \& Niu, Q. Valley-contrasting physics in graphene: magnetic moment and topological transport. Phys. Rev. Lett. 99, 236809 (2007).

5. Shayegan, M. et al. Two-dimensional electrons occupying multiple valleys in AlAs. Phys. Status Solidi B 243, 3629-3642 (2006).

6. Eng, K., McFarland, R. N. \& Kane, B. E. Integer quantum Hall effect on a sixvalley hydrogen-passivated silicon (111) surface. Phys. Rev. Lett. 99, 016801 (2007).

7. Goswami, S. et al. Controllable valley splitting in silicon quantum devices. Nat. Phys. 3, 41-45 (2007). 
8. Pulizzi, F. Spintronics. Nat. Mater. 11, 367-367 (2012).

9. Li, L. et al. Black phosphorus field-effect transistors. Nat. Nanotechnol. 9, 372-377 (2014).

10. Zhu, Z., Collaudin, A., Fauqué, B., Kang, W. \& Behnia, K. Field-induced polarization of Dirac valleys in bismuth. Nat. Phys. 8, 89-94 (2012).

11. Küchler, R. et al. Thermodynamic evidence for valley-dependent density of states in bulk bismuth. Nat. Mater. 13, 461-465 (2014).

12. Ganichev, S. D. et al. Spin-galvanic effect. Nature 417, 153-156 (2002).

13. Stevens, M. J. \& Smirl, A. L. Quantum interference control of ballistic pure spin currents in semiconductors. Phys. Rev. Lett. 90, 136603 (2003).

14. Yao, W., Xiao, D. \& Niu, Q. Valley-dependent optoelectronics from inversion symmetry breaking. Phys. Rev. B 77, 235406 (2008).

15. Xiao, D., Liu, G.-B., Feng, W., Xu, X. \& Yao, W. Coupled spin and valley physics in monolayers of $\mathrm{MoS}_{2}$ and other group-VI dichalcogenides. Phys. Rev. Lett. 108, 196802 (2012).

16. Zhang, Y. J., Oka, T., Suzuki, R., Ye, J. T. \& Iwasa, Y. Electrically switchable chiral light-emitting transistor. Science 344, 725-728 (2014).

17. Mak, K. F., McGill, K. L., Park, J. \& McEuen, P. L. The valley Hall effect in $\mathrm{MoS}_{2}$ transistors. Science 344, 1489-1492 (2014).

18. Yuan, H. et al. Generation and electric control of spin-valley-coupled circular photogalvanic current in WSe 2 . Nat. Nanotechnol. 9, 851-857 (2014).

19. Hofmann, P. h. The surfaces of bismuth: Structural and electronic properties. Prog. Surf. Sci. 81, 191-245 (2006).

20. Koroteev, Y. M. et al. Strong spin-orbit splitting on Bi surfaces. Phys. Rev. Lett. 93, 046403 (2004).

21. Takayama, A., Sato, T., Souma, S. \& Takahashi, T. Giant out-of-plane spin component and the asymmetry of spin polarization in surface rashba states of bismuth thin film. Phys. Rev. Lett. 106, 166401 (2011).

22. Takayama, A., Sato, T., Souma, S., Oguchi, T. \& Takahashi, T. Tunable Spin Polarization in Bismuth Ultrathin Film on Si(111). Nano Lett. 12, 1776-1779 (2012).

23. Ast, C. R. \& Höchst, H. Fermi surface of $\mathrm{Bi}(111)$ measured by photoemission spectroscopy. Phys. Rev. Lett. 87, 177602 (2001).

24. Ast, C. R. \& Höchst, H. Indication of charge-density-wave formation in Bi (111). Phys. Rev. Lett. 90, 016403 (2003).

25. Hirahara, T. et al. Role of spin-orbit coupling and hybridization effects in the electronic structure of ultrathin Bi films. Phys. Rev. Lett. 97, 146803 (2006).

26. Hirahara, T. et al. Quantum well states in ultrathin Bi films: Angle-resolved photoemission spectroscopy and first-principles calculations study. Phys. Rev. B 75, 035422 (2007).

27. Ohtsubo, Y. et al. Giant anisotropy of spin-orbit splitting at the bismuth surface. Phys. Rev. Lett. 109, 226404 (2012).

28. Xiao, S. H., Wei, D. H. \& Jin, X. F. Bi(111) thin film with insulating interior but metallic surfaces. Phys. Rev. Lett. 109, 166805 (2012).

29. Aitani, M. et al. In situ magnetotransport measurements in ultrathin Bi films: evidence for surface-bulk coherent transport. Phys. Rev. Lett. 113, 206802 (2014).

30. Murakami, S. Quantum spin Hall effect and enhanced magnetic response by spin-orbit coupling. Phys. Rev. Lett. 97, 236805 (2006).

31. Liu, Z. et al. Stable nontrivial $Z_{2}$ topology in ultrathin Bi (111) films: a firstprinciples study. Phys. Rev. Lett. 107, 136805 (2011).

32. Wada, M., Murakami, S., Freimuth, F. \& Bihlmayer, G. Localized edge states in two-dimensional topological insulators: Ultrathin Bi films. Phys. Rev. B 83, 121310 (2011).

33. Hirahara, T. et al. Interfacing $2 \mathrm{D}$ and 3D topological insulators: $\mathrm{Bi}$ (111) bilayer on $\mathrm{Bi}_{2} \mathrm{Te}_{3}$. Phys. Rev. Lett. 107, 166801 (2011).

34. Yang, F. et al. Spatial and energy distribution of topological edge states in single Bi (111) bilayer. Phys. Rev. Lett. 109, 016801 (2012).

35. Drozdov, I. K. et al. One-dimensional topological edge states of bismuth bilayers. Nat. Phys. 10, 664-669 (2014).

36. Miao, L. et al. Evolution of the electronic structure in ultrathin Bi (111) films. Phys. Rev. B 91, 205414 (2015).

37. Hasan, M. Z. \& Kane, C. L. Colloquium: topological insulators. Rev. Mod. Phys. 82, 3045-3967 (2010).

38. König, M. et al. Quantum spin Hall insulator state in $\mathrm{HgTe}$ quantum wells. Science 318, 766-770 (2007)

39. Koroteev, Y. M., Bihlmayer, G., Chulkov, E. V. \& Blügel, S. First-principles investigation of structural and electronic properties of ultrathin Bi films. Phys. Rev. $B$ 77, 045428 (2008).

40. Liu, Y. \& Allen, R. E. Electronic structure of the semimetal Bi and Sb. Phys. Rev. B 52, 1566-1577 (1995).

41. Okada, Y. et al. Observation of Dirac node formation and mass acquisition in a topological crystalline insulator. Science 341, 1496-1499 (2013).

42. Miller, D. L. et al. Observing the quantiaztion of zero mass carriers in graphene. Science 324, 924-927 (2009).
43. Van Hove, L. The occurrence of singularities in the elastic frequency distribution of a crystal. Phys. Rev. 89, 1189-1193 (1953).

44. Cheng, P. et al. Landau quantization of topological surface states in $\mathrm{Bi}_{2} \mathrm{Se}_{3}$. Phys. Rev. Lett. 105, 076801 (2010).

45. Hirahara, T. et al. Role of quantum and surface-state effects in the bulk fermilevel position of ultrathin Bi films. Phys. Rev. Lett. 115, 106803 (2015).

46. Hirahara, T. The Rashba and quantum size effects in ultrathin Bi films. J. Electron Spectrosc. Relat. Phenom. 201, 98-104 (2015).

47. Zhang, H.-J. et al. Electronic structues and surface states of the topological insulator $\mathrm{Bi}_{1-x} \mathrm{Sb}_{x}$. Phys. Rev. B 80, 085307 (2009).

48. Hsieh, D. et al. Observation of unconventional quantum spin textures in topological insulators. Science 323, 919-922 (2009).

49. Souma, S. et al. Direct measurement of the out-of-plane spin texture in the dirac-cone surface state of a topological insulator. Phys. Rev. Lett. 106, 216803 (2011).

50. Zhu, Z., Fauqué, B., Fuseya, Y. \& Behnia, K. Angle-resolved Landau spectrum of electrons and holes in bismuth. Phys. Rev. B 84, 115137 (2011).

51. Hashimoto, K. et al. Quantum Hall transition in real space: from localized to extended states. Phys. Rev. Lett. 101, 256802 (2008).

52. Fuseya, Y. et al. Origin of the large anisotropic $g$ factor of holes in Bismuth. Phys. Rev. Lett. 115, 216401 (2015).

53. Hirahara, T. et al. Origin of the surface-state band-splitting in ultrathin Bi films: from a Rashba effect to a parity effect. New J. Phys. 10, 083038 (2008).

54. Komnik, Y. F. \& Andrievskii, V. V. Kinetic properties of bismuth thin films. Thin Solid Films 1, 51-58 (1975).

55. Hoffman, C. A. et al. Semimetal-to-semiconductor transition in bismuth thin films. Phys. Rev. B 48, 11431-11434 (1993).

56. Hirahara, T. et al. Large surface-state conductivity in ultrathin Bi films. Appl. Phys. Lett. 91, 202106 (2007).

57. Ast, C. R. \& Höchst, H. Two-dimensional band structure and self-energy of Bi (111) near the $\bar{\Gamma}$ point. Phys. Rev. B 66, 125103 (2002).

58. Seradjeh, B., Wu, J. S. \& Phillips, P. Signatures of surface states in Bismuth at high magnetic fields. Phys. Rev. Lett. 103, 136803 (2009).

59. Sadowski, J. et al. Thin bismuth film as a template for pentacene growth. Appl. Phys. Lett. 86, 073109 (2005).

60. Blaha, P., Schwarz, K., Madsen, G. K. H., Kvasnicka, D. \& Luitz, J. WIEN2k, An Augmented Plane Waves Plus Local Orbitals Program for Calculating Crystal Properties (Karlheinz Schwarz, Technische Universität Wien, 2001).

61. Perdew, J. P., Burke, K. \& Emzerhof, M. Generalized gradient approximation made simple. Phys. Rev. Lett. 77, 3865-3868 (1996).

\section{Acknowledgements}

This work was financially supported by the Ministry of Science and Technology of China (grants 2011CB921400), the 'Strategic Priority Research Program' of the Chinese Academy of Sciences (grant XDB01020100), and the National Natural Science Foundation of China (grants 91321309, 91421313, 51132007, 21421063 and 11274284).

\section{Author contributions}

B.W. and J.G.H. designed the research; H.J.D., X.G.L., J.F.W., M.Y.T. performed the experiments; X.S. performed theoretical calculations and analysis, and B.W., X.J.W., A.D.Z., Y.L. and J.L.Y. analysed the data; B.W. and Y.L. wrote the manuscript. All authors contributed to writing and revising the manuscript. H.J.D. and X.S. contributed equally to this work

\section{Additional information}

Supplementary Information accompanies this paper at http://www.nature.com/ naturecommunications

Competing financial interests: The authors declare no competing financial interests

Reprints and permission information is available online at http://npg.nature.com/ reprintsandpermissions/

How to cite this article: Du, H. et al. Surface Landau levels and spin states in bismuth (111) ultrathin films. Nat. Commun. 7:10814 doi: 10.1038/ncomms10814 (2016).

This work is licensed under a Creative Commons Attribution 4.0 International License. The images or other third party material in this article are included in the article's Creative Commons license, unless indicated otherwise in the credit line; if the material is not included under the Creative Commons license, users will need to obtain permission from the license holder to reproduce the material. To view a copy of this license, visit http://creativecommons.org/licenses/by/4.0/ 